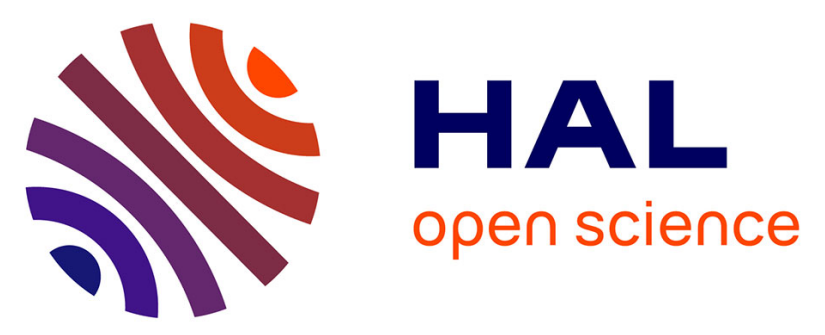

\title{
Brief intervention in substance-use among adolescent psychiatric patients: a randomized controlled trial
}

Javier Goti, Rosa Diaz, Lourdes Serrano, Laura Gonzalez, Rosa Calvo, Antoni Gual, Josefina Castro

\section{- To cite this version:}

Javier Goti, Rosa Diaz, Lourdes Serrano, Laura Gonzalez, Rosa Calvo, et al.. Brief intervention in substance-use among adolescent psychiatric patients: a randomized controlled trial. European Child and Adolescent Psychiatry, 2009, 19 (6), pp.503-511. 10.1007/s00787-009-0060-5 . hal-00535176

\section{HAL Id: hal-00535176 https://hal.science/hal-00535176}

Submitted on 11 Nov 2010

HAL is a multi-disciplinary open access archive for the deposit and dissemination of scientific research documents, whether they are published or not. The documents may come from teaching and research institutions in France or abroad, or from public or private research centers.
L'archive ouverte pluridisciplinaire HAL, est destinée au dépôt et à la diffusion de documents scientifiques de niveau recherche, publiés ou non, émanant des établissements d'enseignement et de recherche français ou étrangers, des laboratoires publics ou privés. 


\title{
Brief intervention in substance-use among adolescent psychiatric patients: a randomized controlled trial
}

\author{
Javier Goti $\cdot$ Rosa Diaz $\cdot$ Lourdes Serrano . \\ Laura Gonzalez $\cdot$ Rosa Calvo $\cdot$ Antoni Gual · \\ Josefina Castro
}

Received: 10 February 2009/Accepted: 9 September 2009/Published online: 25 September 2009

(C) Springer-Verlag 2009

\begin{abstract}
Objective of the study is to assess the efficacy of a brief motivational enhancement intervention in adolescents referred to psychiatric treatment who reported substance-use. In a sample of adolescents $(n=237)$ consecutively admitted to a psychiatry department, 143 were identified as users. Subjects were randomly allocated to one of two groups: an experimental group that received a brief intervention aimed at increasing their awareness of the risks of substance-use, or a control group. All subjects received standard treatment according to the primary diagnosis. Structured questionnaires assessing knowledge, problems, perception of risks and intention of use of psychoactive substances were administered upon admission and 1 month later. Fifty-nine subjects entered the experimental group and 44 the control group. No significant differences between the two groups were identified in socio-demographic features or substance-use. Non-
\end{abstract}

J. Goti $(\bowtie) \cdot$ R. Diaz · L. Serrano · L. Gonzalez · R. Calvo ·

J. Castro

Department of Child and Adolescent Psychiatry and Psychology, Centro de Investigación Biomédica en Red de Salut Mental (CIBER-SAM Network), Hospital Clinic Barcelona,

Barcelona, Spain

e-mail: jgoti@clinic.ub.es

A. Gual

Alcohol Unit, Neurosciences Institute,

Hospital Clinic Barcelona, Barcelona, Spain

J. Castro

IDIBAPS (Institut d'Investigacions Biomèdiques August Pi i

Sunyer), Hospital Clinic Barcelona, Barcelona, Spain

J. Castro

Department of Psychiatry and Clinical Psychobiology,

University of Barcelona, Hospital Clinic Barcelona,

Barcelona, Spain parametric analyses showed a significant increase across time in overall knowledge about drugs and perception of risk in the experimental group $(P<0.05)$. A significant increase in overall knowledge in the experimental group compared to controls was found $(P<0.05)$. No differences were observed for other variables such as intention of use or perception of risk. Brief intervention in adolescents entering psychiatric treatment led to a significant change in overall knowledge about psychoactive substances but not in other variables related to use. Our results point to the need of more intensive interventions.

Keywords Adolescent - Substance-use . Brief intervention - Child psychiatry

\section{Introduction}

According to data from the Spanish Drugs Observatory over a 10-year period (1994-2004) a twofold increase in the prevalence of recent cannabis use (last 30 days) and a threefold increase in the prevalence of recent cocaine use among adolescents aged 14-18 has been reported. Furthermore, a decrease in the age of first use has been reported [26]. A limited knowledge of drug effects and subsequent low perception of risks related to substance-use has been noted as a factor (among others) that might contribute to these changes in substance-use among adolescents [24]. Research on substance-use disorders has stressed the importance of prevention and early intervention among adolescents to improve the awareness of substance-use risks. In this regard, a meta-analysis of preventive school-based programs has shown that those based on interactive rather than directive techniques are more effective [29]. 
Motivational interviewing (MI) is a style of behavior change counseling developed originally to help people change substance-abuse behaviors [18]. The principles of MI include understanding the client's view, avoiding resistances by means of a non-confrontational approach and increasing clients' self-efficacy and the perceived discrepancy between their behavior and their current and long-term goals. Over the years this model of intervention has been shown to be effective in enhancing motivation towards change in several health-related behaviors [8]. Furthermore, some authors have stressed the importance of capitalizing on a teachable moment or window of opportunity [3], which provides good reason to conduct interventions in medical settings such as emergency rooms after a consultation related to substance-use. To implement such interventions, short therapeutic consultations delivered according to the principles of MI have been developed. These approaches, called brief interventions (BI), usually last 1-5 sessions and include several stages: assessment, feedback, information, advice and providing self-help materials [4]. BI have been shown to be effective in reducing substance-use, particularly among non-dependent subjects, attaining a good cost-effectiveness relationship [20]. Studies have yielded some positive results for brief interventions aimed at decreasing substance-use among adolescents in different settings, such as emergency rooms or outpatient settings (general practitioners, pediatric outpatient units) and even in high schools $[10,13,27]$. An important issue when considering brief interventions is the targeted population. Interventions in vulnerable groups such as those already showing drug-related health problems have been tested; Tait et al. [28] demonstrated the effectiveness of a brief intervention with adolescents who were seen in hospital emergency departments for presentations related to alcohol use, for instance. However, one of the socalled high-risk adolescent populations, those who present a comorbid psychiatric condition, has been less investigated. Prevalence of use, abuse and dependence in adolescent psychiatric population has been reported to be high [21], and comorbid psychiatric disorders among adolescents with substance disorders are highly prevalent, with rates reaching $88 \%$ [6].

The objective of the present study was to assess the short-term efficacy of a brief intervention that aimed to change attitudes and thoughts about substance-use among adolescent substance users who were undergoing psychiatric or psychological treatment because of another disorder not primarily related to substance-use. In light of reports which consider that brief interventions capitalizing on a teachable moment are more likely to be effective, we predicted that these adolescents attending a psychiatric unit for non-drug related disorders would be receptive to such an intervention and more willing to change.

\section{Methods}

\section{Subjects}

The present study formed part of an epidemiological and treatment study of the substance-use behaviors of a cohort of adolescents entering psychiatric treatment. Adolescents aged 12-17 years consecutively referred for psychiatric or psychological assessment and treatment to a Child and Adolescent Psychiatry and Psychology Department completed an assessment protocol that included questions regarding their substance-use. Patients who refused to participate $(n=48)$, those who did not complete a substantial part of the protocol $(n=33)$, those $(n=10)$ who suffered from acute psychopathological disturbances (psychotic state, severe depression), and patients who presented mental retardation $(n=15)$ were excluded. Another six patients were referred to a residential center for a more intensive intervention before finishing the evaluation protocol and were consequently not included in this study. Four of them presented a severe substance-use disorder. Finally, 237 individuals and their tutors completed the baseline evaluation protocol and were included in the epidemiological study. Those patients who reported substance-use (according to the study criteria, see below) were considered eligible for the present study and were invited to take part in the treatment trial.

\section{Procedure}

The epidemiological and the intervention study were explained to the adolescents and their parents or mentors, and written informed consent was obtained. Subjects were then assessed with a test battery in accordance with a protocol approved by the Ethics Committee of the institution. Whenever substance-use was identified, subjects who were willing to participate in the intervention study were randomly allocated to one of two possible conditions: an intervention group in which, after completion of the baseline evaluation, both the adolescent and his/her parents each received one separate individual session; and a control group [treatment as usual (TAU)] in which no further intervention was delivered. Control group will be referred from now on and throughout this paper as TAU group. Probands in both groups entered the protocol at the beginning of the treatment in the department, not having received previous treatment in our service. After inclusion and baseline evaluation was completed, subjects in both the intervention and the TAU group received standard care according to the established diagnoses and the intervention schedule of the Child and Adolescent Psychiatry and Psychology Department. TAU comprised diagnostic evaluation according to the presenting problem, and an initial 
therapeutic approach, either pharmacological and/or cognitive-behavioral therapy. The observation period for all probands undertook 1 month, thus including no more than 2-4 sessions. Therefore, most of TAU consisted of the completion of the initiated diagnostic evaluation and general therapeutic recommendations on behalf of the evolving psychiatric diagnoses. Follow-up visits took place at 1 month and included a reevaluation of part of the study variables. Follow-up evaluators were blind to the condition of intervention or TAU group.

\section{Study variables}

\section{Socio-demographic characteristics and psychiatric diagnosis}

Children were evaluated via semi-structured interviews based on those used in the Collaborative Studies on Genetics of Alcoholism (COGA) Project [11], which were translated and adapted into Spanish. These interviews provided measures of socio-demographic variables: age, gender, socioeconomic status, psychiatric status and school achievement, among other variables. An initial diagnosis was determined according to DSM-IV [1]. Primary diagnoses were grouped into five possible categories: mood disorders, anxiety disorders, adjustment disorders, externalizing disorders (including ADHD and conduct disorders) and eating disorders. Comorbid diagnoses were explored and ascertained.

\section{Substance-use pattern}

Quantity and frequency measures of substance-use were obtained from the semi-structured interview and patients' clinical records. In order to improve the assessment, patients were not required to disclose their drug use to their parents. Accordingly, the pattern of use of tobacco, alcohol, cannabis and other substances (basically, cocaine, amphetamines and designer drugs) was coded into five categories as follows: 1, no use; 2, occasional use: from time to time, at parties, during holidays or social events; 3, regular use: almost daily use for tobacco, almost weekly use for alcohol and cannabis, almost monthly use for stimulants or other drugs, with no clear evidence of drug-related problems; 4, substance-use problems (SUP): quantity-frequency and/or situational pattern of use with a high probability of developing health or psycho-social problems, now or in the future, but still sub-diagnostic; and 5, diagnosis of abuse or dependence according to DSM-IV-TR criteria. Subjects were also interviewed using a Spanish version of the Teen Addiction Severity Index (T-ASI) [7], a semistructured interview adapted to adolescents from the
Adult Addiction Severity Index (ASI) [12]. The version used in this study includes 142 items that assess problems usually related with drug use in seven domains. Severity of problems in each domain is scored on a five-point scale (0-4) according to both patient subjective criteria and interviewer criteria. For analytic purposes we divided the sample into two groups according to scores on the Teen ASI drug subscale, each group including subjects with either minor to moderate $(0-2)$ versus severe problems (3-4) with drugs.

\section{Variables related to use}

Structured questionnaires were employed to assess several areas of interest regarding subjects' thoughts about psychoactive substances. Questionnaires were obtained from the Spanish version of the Evaluation Instrument Bank (EIB) and from the European Monitoring Centre for Drugs and Drug Addiction (EMCDDA) [14], and were used to assess the following areas:

Knowledge about psychoactive substances A 31-item questionnaire that assesses whether the subject has accurate knowledge about the effects of psychoactive substances (e.g., "alcohol is a stimulant substance").

Risk perception A 13-item questionnaire to be rated on a 4-point response scale and which evaluates the subjects' view of the potential harm related to the use of different substances.

Problems derived from use An 8-item questionnaire to be scored on a 4-point scale (never, 1 or 2 times, 3 or 4 times, more than 5 times) and which enquires about the past presentation of drug-related problems and psychosocial consequences (e.g., being ill, fights, legal problems, etc.).

Intention to use In this questionnaire the probability of using several psychoactive substances is scored on a 5point scale (1, "highly probable use" to 5, "very improbable use"). Higher scores in this questionnaire indicate a lower probability of use.

Intervention

\section{Intervention group}

After completion of baseline evaluation, patients allocated to the intervention group received a brief therapeutic intervention designed according to the standards of motivational interviewing and adapted from previous work on this topic [15]. The intervention consisted of: 
- An individual session with the adolescent. This interview lasted approximately $60 \mathrm{~min}$. Materials related to the interview were developed according to previous reports on the subject [15-17]. The mentioned intervention had been previously manualized by the original authors, contacted by the authors, and it was translated and adapted to the Spanish language. The intervention considered altogether 12 points to be discussed during the session: (1) contact, (2) feedback from the evaluation, (3) analysis of an episode of substance-use, (4) pros and cons of substance-use, (5) personal goals, (6) problems and risks of substance-use, (7) explore preoccupations, (8) making decisions, (9) questions and answers, (10) decisional balance, (11) planning changes, (12) self-monitoring. Through this planned structure therapists focused initially in building a good rapport, and the interviewer involved the patient in an initial discussion about the results of the evaluation. This led to a review of the drugs used by the subject and an elicitation of positives and negatives of drug use. According to the particular areas of interest reflected by the subject, the relationship between drug use and current and long-term goals was explored. Possible discrepancies and hypothetical problems in the future related to substance-use were examined, and information and counseling was offered. A decisional balance was developed and, if accepted by the subject, advice and exploration of suitable tools to plan changes in substance-use were offered. At each point, the basic components of the motivational interview approach (empathy, non-confrontation, acceptance and support of self-efficacy and autonomy) were contemplated and several techniques and skills were planned [e.g., (1) contact: open questions; (4) pros and cons: Open questions, reflective listening, and pointing dissonance].

- An individual session with parents or mentors. This interview consisted in the presentation of educational materials and a brief counseling intervention on parenting skills and adolescent substance-use, its consequences and the relevance of monitoring and intervention.

Training on the intervention was provided by a doctoral level supervisor experienced in motivational interviewing training. Training consisted in over $20 \mathrm{~h}$ session time that included role-playing and direct supervision of the implementation of motivational interviewing techniques and skills and the adherence to the manualized intervention. All interventions were conducted by the same two psychologists with significant clinical experience in treating substance-use disorders. During the trial, at least $15 \%$ of the performed interventions were supervised by external observers and feedback was provided to the interviewers by the supervisor.

\section{Treatment as usual (TAU)}

Individuals assigned to this group and their parents or tutors received standard care and no further intervention other than completion of the assessment protocol. As described previously, standard care consisted of psychiatric and psychological examination and interviewing with both the parents and the patients. Diagnostic procedures and therapeutic recommendations were delivered on behalf of the evolving diagnoses, and were naturalistically set by the professionals in the department according of the practice guidelines of the department. Visits were offered in a 1-2 week basis.

\section{Statistical analyses}

Baseline variables in the two groups were compared for equivalence. Categorical variables were analyzed with $\chi^{2}$ and numerical variables by means of the Student's $t$ test.

To assess short-term changes in the several variables related to drug use, the scores on the psychometric scales at baseline and at 1-month follow-up were analyzed as the main outcome variable. Study variables were first checked for distributional assumptions by means of the Kolmogorov -Smirnov test. Due to skewness in the distribution of variables, the Wilcoxon signed-rank test was used to compare mean scores on the scales between baseline and the endpoint (1 month) evaluation in the two groups. Furthermore, a univariate analysis of variance with baseline scores as the covariate (ANCOVA) was performed to compare differences in scores on the psychometric scales between the two groups. Data were analyzed using an intention to treat approach.

\section{Results}

One hundred and forty-three adolescents $(60.3 \%)$ of the 237 who completed the baseline evaluation were identified as substance users, with five of them qualifying for substance abuse or dependence (other than nicotine) according to the study criteria. Seventy-eight individuals were allocated to the intervention group; nine subjects refused to participate in the brief intervention trial and ten subjects were lost to follow-up. Sixty-five individuals were assigned to the TAU group; 21 out of them were lost to follow-up (see Fig. 1). Follow-up interviews were conducted at 1 month. Finally, 59 in the intervention group and 44 in the TAU were included in the analysis. The mean age of 


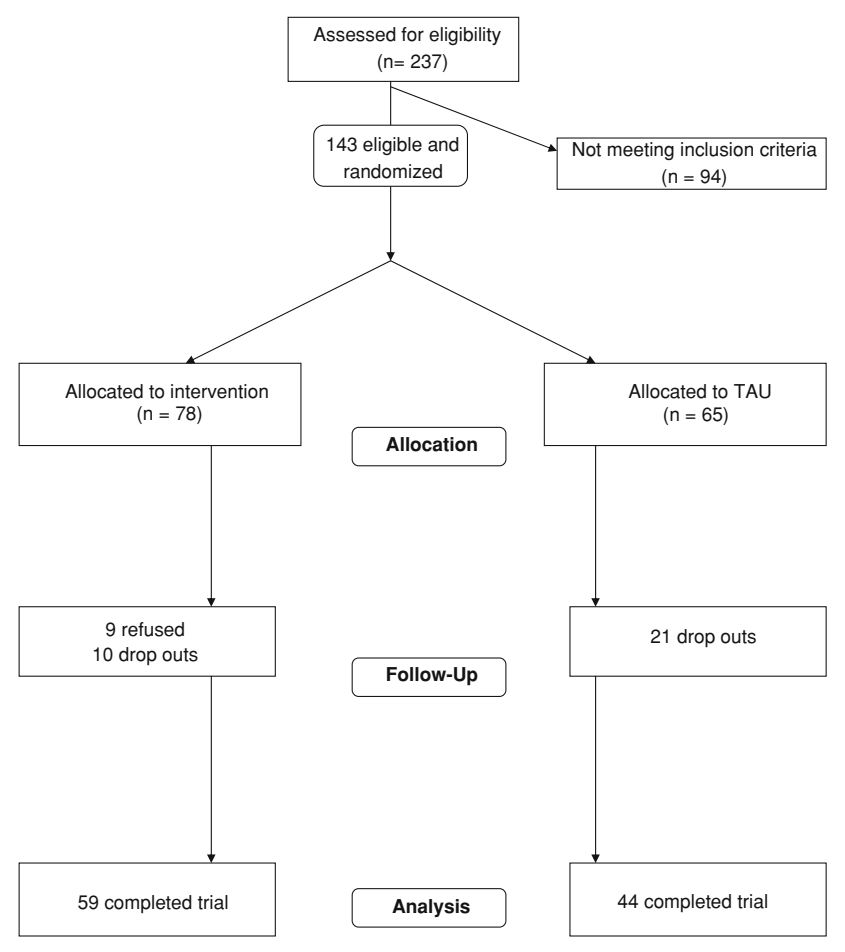

Fig. 1 Patient flowchart across study

participants was 15.2 years (SD 1.2) with a range of 12-17 years; $75.7 \%(n=78)$ of participants were female.

Socio-demographic characteristics of the participants in the two groups, including age, gender distribution, socioeconomic status, main and comorbid psychiatric diagnosis are shown in Table 1. Substance-use indicators, including age of onset and average frequency of use among participants in both groups are shown in Table 2. No significant differences were found between the two randomized groups in terms of socio-demographic features. A trend towards a difference in baseline scores in the drug subscale of the T-ASI between the two groups was found $(t=-1.9$; $P=0.06)$ though it did not reach statistical significance. According to scores on the T-ASI drug subscale, subjects were divided into two groups, each one including subjects with either minor to moderate (0-2) versus severe problems (3-4) with drugs. Percentage of subjects reporting severe problems were not significantly different between the two groups.

The Wilcoxon signed-rank test revealed a significant change over time (1 month after baseline assessment) in scores on the overall knowledge of psychoactive substances questionnaire in both the TAU group $(z=-2.08$; $P=0.03)$ and the intervention group $(z=-4.08$; $P=0.0001)$. Furthermore, there was a significant change in perception of risks after the intervention among participants in the experimental group $(z=-1.97 ; P=0.04)$ but not among subjects in the TAU group $(z=-0.85$;
Table 1 Socio-demographic characteristics, school achievement and psychiatric diagnosis

\begin{tabular}{|c|c|c|c|c|}
\hline & $\begin{array}{l}\text { Intervention } \\
(n=59)\end{array}$ & $\begin{array}{l}\text { TAU } \\
(n=44)\end{array}$ & $\begin{array}{l}\text { Statistic } \\
\text { test }\end{array}$ & $\begin{array}{l}P \\
\text { value }\end{array}$ \\
\hline \multicolumn{5}{|l|}{ Age } \\
\hline Mean (SD) & $15.4(1.2)$ & $15.1(1.2)$ & $t=-$ & $0.16^{\mathrm{a}}$ \\
\hline $12-13$ years $[N(\%)]$ & $3(5.1)$ & $3(6.7)$ & $\chi^{2}=4.4$ & $0.11^{\mathrm{b}}$ \\
\hline $14-15$ years $[N(\%)]$ & $25(42.4)$ & $27(61.5)$ & & \\
\hline $16-17$ years $[N(\%)]$ & $31(52.5)$ & $14(31.8)$ & & \\
\hline \multicolumn{5}{|l|}{ Gender } \\
\hline Male $[N(\%)]$ & $18(30.5)$ & $7(15.9)$ & $\chi^{2}=2.92$ & $0.1^{\mathrm{b}}$ \\
\hline Female $[N(\%)]$ & $41(69.5)$ & $37(84.1)$ & & \\
\hline \multicolumn{5}{|c|}{ Socioeconomic status $N(\%)$} \\
\hline Low & $1(1.7)$ & $1(2.2)$ & $\chi^{2}=0.74$ & $0.68^{\mathrm{b}}$ \\
\hline Medium & $54(91.6)$ & $40(90.9)$ & & \\
\hline High & $4(6.7)$ & $3(6.8)$ & & \\
\hline \multicolumn{5}{|l|}{ Main diagnosis $N(\%)$} \\
\hline Mood disorders & $8(13.5)$ & $4(9.1)$ & $\chi^{2}=4.4$ & $0.35^{\mathrm{b}}$ \\
\hline Eating disorder & $22(37.4)$ & $25(56.9)$ & & \\
\hline $\begin{array}{l}\text { Externalizing } \\
\text { disorder }\end{array}$ & $18(30.5)$ & $11(25)$ & & \\
\hline Anxiety disorders & $5(8.5)$ & $2(4.5)$ & & \\
\hline $\begin{array}{l}\text { Adjustment } \\
\text { disorders }\end{array}$ & $6(10.1)$ & $2(4.5)$ & & \\
\hline \multicolumn{5}{|l|}{ Comorbid diagnosis } \\
\hline $\begin{array}{l}\text { No comorbid } \\
\text { diagnosis }\end{array}$ & 38 & 26 & $\chi^{2}=5.1$ & 0.4 \\
\hline Mood disorder & 5 & 10 & & \\
\hline Conduct disorder & 6 & 3 & & \\
\hline Anxiety disorder & 4 & 3 & & \\
\hline $\begin{array}{l}\text { Adjustment } \\
\text { disorders }\end{array}$ & 2 & 1 & & \\
\hline $\begin{array}{l}\text { substance-use } \\
\text { disorder }\end{array}$ & 4 & 1 & & \\
\hline \multicolumn{5}{|c|}{ School achievement $[N(\%)]$} \\
\hline Low & $19(32.2)$ & $15(34.1)$ & $\chi^{2}=0.51$ & $0.97^{\mathrm{b}}$ \\
\hline Medium & $29(49.1)$ & $21(47.7)$ & & \\
\hline High & $11(18.7)$ & $8(18.2)$ & & \\
\hline
\end{tabular}

SUD Substance-use related disorders, TAU treatment as usual, $S D$ standard deviation

a Student's $t$

b Chi-square

$P=0.39)$. Scores on the Problems with drugs scale and the Intention to use scale did not change significantly over time among participants in either of the two groups (see Table 3).

To further assess differences between the two groups as regards short-term changes (1 month) in scores on the psychometric scales used, we performed a group analysis of variance with baseline scores on the scales as a covariate 
Table 2 Substance-use parameters at baseline
$S D U$ Standard drink units, TAU treatment as usual, $S D$ standard deviation

a Student's $t$

${ }^{b}$ Chi-square

\begin{tabular}{|c|c|c|c|c|}
\hline & $\begin{array}{l}\text { Intervention } \\
(n=59)\end{array}$ & $\begin{array}{l}\text { TAU } \\
(n=44)\end{array}$ & $\begin{array}{l}\text { Statistic } \\
\text { test }\end{array}$ & $P$ value \\
\hline \multicolumn{5}{|l|}{ Alcohol [mean (SD)] } \\
\hline Monthly average SDU & $3.4(4.6)$ & $3.8(6)$ & $t=-0.28$ & $0.78^{\mathrm{a}}$ \\
\hline Episodes of use $>5$ SDU & $5.5(15.5)$ & $7.2(19.9)$ & $t=0.38$ & $0.7^{\mathrm{a}}$ \\
\hline Age onset & $13.4(1.7)$ & $13.3(1.4)$ & $t=-0.41$ & $0.67^{\mathrm{a}}$ \\
\hline \multicolumn{5}{|l|}{ Tobacco [mean (SD)] } \\
\hline Average cigarettes/week & $50.9(65.7)$ & $51.5(63.6)$ & $t=-0.85$ & $0.4^{\mathrm{a}}$ \\
\hline Age onset & $12.4(2.0)$ & $12.4(1.8)$ & $t=-0.14$ & $0.88^{\mathrm{a}}$ \\
\hline \multicolumn{5}{|l|}{ Cannabis [mean (SD)] } \\
\hline Average "joints"/week & $5.6(10.8)$ & $5.8(16.4)$ & $t=-1.16$ & $0.24^{\mathrm{a}}$ \\
\hline Age onset & $13.7(1.3)$ & $13.8(1.5)$ & $t=0.56$ & $0.57^{\mathrm{a}}$ \\
\hline \multicolumn{5}{|l|}{ Other drugs [mean (SD)] } \\
\hline Episodes of use & $3.9(14.8)$ & $3.6(16.9)$ & $t=-0.34$ & $0.72^{\mathrm{a}}$ \\
\hline Age onset & $14.9(1.1)$ & $14.8(15)$ & $t=-0.26$ & $0.79^{\mathrm{a}}$ \\
\hline $\begin{array}{l}\text { Baseline scores in T-ASI } \\
\text { drug subscale [mean (SD)] }\end{array}$ & $2.3(0.95)$ & $2.09(1.09)$ & $t=-1.9$ & $0.06^{\mathrm{a}}$ \\
\hline \multicolumn{5}{|l|}{$\begin{array}{l}\text { Substance-use severity } \\
\text { (according to T-ASI) }\end{array}$} \\
\hline Minor problems $[N(\%)]$ & $38(64)$ & $34(77)$ & $\chi^{2}=2.57$ & $0.12^{\mathrm{b}}$ \\
\hline Severe problems $[N(\%)]$ & $21(36)$ & $10(22)$ & & \\
\hline $\begin{array}{l}\text { Prevalence of regular substance } \\
\text { users (other than tobacco) }[N(\%)]\end{array}$ & $42(71)$ & $25(56)$ & $\chi^{2}=2.28$ & $0.14^{\mathrm{b}}$ \\
\hline
\end{tabular}

Table 3 Changes in drug-scale scores at 1-month follow-up

\begin{tabular}{|c|c|c|c|c|c|c|c|c|c|c|c|c|}
\hline & \multicolumn{4}{|c|}{ Intervention $(n=59)$} & \multicolumn{4}{|c|}{ TT as usual $(n=44)$} & \multicolumn{2}{|c|}{$\begin{array}{l}\text { Group } \\
\text { differences }\end{array}$} & \multicolumn{2}{|c|}{$\begin{array}{l}\text { Group } \\
\text { differences }\end{array}$} \\
\hline & $\begin{array}{l}\text { Baseline [mean } \\
(\mathrm{SD})]\end{array}$ & $\begin{array}{l}1 \text { month [mean } \\
(\mathrm{SD})]\end{array}$ & $z$ & $P$ & $\begin{array}{l}\text { Baseline [mean } \\
\text { (SD)] }\end{array}$ & $\begin{array}{l}1 \text { month [mean } \\
(\mathrm{SD})]\end{array}$ & $z$ & $P$ & $F$ & $P$ & $F$ & $P$ \\
\hline Knowledge & $16.9(4.8)$ & $19.6(4.8)$ & 4.08 & $0.0001^{\mathrm{a}}$ & $16.1(4.2)$ & $17.2(4.5)$ & 2.08 & $0.03^{\mathrm{a}}$ & 6.25 & $0.01^{\mathrm{b}}$ & 9.63 & $0.003^{\mathrm{c}}$ \\
\hline Problems & $11.7(4.2)$ & $11.8(4.2)$ & 1.05 & 0.29 & $10.7(4.1)$ & $10.9(3.4)$ & 0.61 & 0.54 & 0.72 & 0.39 & 0.27 & 0.6 \\
\hline $\begin{array}{l}\text { Intention to } \\
\text { use }\end{array}$ & $37.7(7)$ & $37.5(5.1)$ & 0.62 & 0.53 & $36.8(5.1)$ & $37.9(6.5)$ & 0.83 & 0.4 & 0.97 & 0.32 & 0.44 & 0.5 \\
\hline $\begin{array}{l}\text { Perception of } \\
\text { risk }\end{array}$ & $39.7(8.2)$ & $41.8(6.1)$ & 1.97 & $0.04^{\mathrm{a}}$ & $40(7.9)$ & $40.1(7.7)$ & 0.85 & 0.39 & 2.24 & 0.137 & 3.24 & 0.67 \\
\hline
\end{tabular}

TT as usual Treatment-as-usual group, $S D$ standard deviation

${ }^{a}$ Wilcoxon signed-rank test

b Analysis of variance with baseline score as covariate (ANCOVA), mean difference between baseline and endpoint scores as the dependent variable, and allocation to intervention or TAU group as the grouping factor

${ }^{c}$ Analysis of variance with baseline score and baseline severity of use as covariates (ANCOVA), mean difference between baseline and endpoint scores as the dependent variable, and allocation to intervention or TAU group as the grouping factor

(ANCOVA), the mean difference between baseline and endpoint scores as the dependent variable, and allocation to intervention or TAU group as the grouping factor. This analysis revealed a significant difference between the two groups in the mean difference between baseline and endpoint scores on the questionnaire measuring overall knowledge about psychoactive substances $(F=6.25$;
$P=0.01)$. None of the differences between the two groups in their scores on the Problems with Drugs, Intention to Use or Perception of Risks scales reached statistical significance (see Table 3). Since a trend towards a difference between both groups in the severity of baseline use was found, to assess the possible influence of baseline substance-use in the group differences we performed another 
ANCOVA, with the same parameters but adding the severity of use according to baseline T-ASI scores as covariate. A significant difference between the two groups in the scores on the Knowledge scale persisted $(F=9.6$; $P=0.003)$. No group differences in the rest of the scales were found (Problems, Intention of Use, Perception of Risk).

\section{Discussion}

In a group of patients attending a psychiatry and psychology department, the BI resulted in a significant increase in knowledge about the effects of psychoactive substances and an increased awareness of their implicit risks. The TAU group also experienced an increase in their knowledge, but the difference between the two groups reached statistical significance and favored the experimental group. When comparing the results in both groups considering baseline scores as a covariate, the difference in risk perception lost its significance. No other significant differences between the two conditions were found for the other outcome variables, and intention to use did not differ over time between the two groups.

Perception of risks has been proposed as a factor linked to the likelihood of substance use among adolescents [24]; the more favorable the attitude of young people towards substance-use, the lower the perception of risk and the more mistaken their beliefs [19]. Thus, increased knowledge about the real effects of psychoactive substances and a better perception of the risks involved can be regarded as a significant beneficial effect of the intervention. However, other authors have failed to confirm that risk perception is a core indicator of substance-use [30], and argue that perceived pleasure or benefits constitute a more significant predictor of the intention and frequency of use.

Though an increase in the knowledge is regarded a positive outcome, the small change in the psychometric scale scores, coupled with the lack of concurrent changes in the intention of use, all points towards an undermined effect of the brief intervention in this sample.

There may be several explanations for these results. Our original prediction was that adolescents attending a psychiatric unit for non-drug related disorders would be receptive to such an intervention. In contrast, it could be that our studied population is less likely to benefit from such an intervention. The sample consisted of adolescents who already present with a primary psychiatric diagnosis that have led to the need for a mental examination, and such individuals represent a subgroup at risk of developing substance-use problems. However, these subjects might also present a greater lack of insight due to their psychopathology. Lack of insight is both one of the targets of motivational interviewing, as well as one of the factors that contribute to resistance. It has been shown that among adult psychiatric patients with comorbid substance-use disorders, motivational interviewing, although feasible, only yields modest and short-term effects, and more extensive interventions might therefore be recommended [2].

On the other hand, it has been previously noted, at least for alcohol disorders, that BI are less effective among those subjects with substance dependence than for those with less severe substance-use problems [5]. This suggests that BI techniques may be an appropriate form of intervention with adolescents who present less entrenched substance-use behaviors, whereas such an intervention is too short for those with more severe problems. We tested this hypothesis in our trial and found that baseline level of use, coded in two categories according to scores on the teen ASI drug subscale [minor to moderate (0-2) vs. severe problems (3-4) with drugs], did not significantly influence the difference between both groups (intervention vs. TAU).

Nevertheless, it should be borne in mind that defining the severity of substance-use among adolescents using a categorical approach is questionable and might not fit well with our data. Debates on the conceptualization of disorders arising from the use of cannabis (and other substances) among adolescents question the current dichotomy between cannabis abuse and dependence [23]. Some authors propose a dimensional approach towards cannabisuse disorders in adolescents that could possibly yield more appropriate predictive power in terms of prognosis and therapeutic needs [9].

Age also appears to be an important predictor of treatment outcome in BI, as shown by data from a meta-analytic review of the efficacy of these interventions [31]. Shakeshaft et al. found that a brief motivational intervention was more efficacious among clients who were older at baseline, indicating that older participants in their study were more active in treatment and less likely to withdraw. Adolescent patients might thus be less receptive to such an intervention [25].

Another possible explanation would be that the intervention was insufficient, either in its quality or quantity. Our protocol design considered the need to ensure the quality of the intervention provided, and a significant effort in manualizing and training was done. The interviewers adhered well to the principles of motivational interviewing, however, we are aware that the absence of an objective measurement of the supervision performed of the delivered intervention during the trial represents a shortcoming. Thus, a possible qualitative lack in the delivered intervention, though less likely in our view, must be considered as a potential explanation to our results. The "amount" of therapy offered was established on the basis of previous 
reports of BI in adolescents [15], and once again the design adhered well to the model of BI. A possible shortcoming could be related to the fact that although BI have proven to be efficient in previous controlled trials, most of these studies have focused on specific behaviors such as cigarette smoking or alcohol use. In contrast, studies reporting on BI oriented toward broader aspects (e.g., substance-use as a whole, considering several different substances) have not reported the same efficacy [16].

It might also be asked whether the chosen outcome measures constitute a good measure of the benefits generated by our intervention. The selected psychometric scales are included in a comprehensive bank of standardized instruments that provide valuable information on subjective features related to adolescents' substance-use. However, we are aware that these scales have not been designed for prospective assessment in the context of an intervention trial. Another limitation derives from the fact that no biological test (e.g., urinary or saliva drug test) was performed at baseline to ensure the accuracy of the selfreport. Nevertheless, substance-use assessment by means of self-report questionnaires and direct interviews with adolescents has been proven to be efficient in previous studies [22].

Further limitations could rely on the fact that our sample consisted of $75.7 \%$ of female participants. This resulted from a nonstratified recruiting procedure (subjects were included consecutively) with our department having substantially increased number of female adolescents, since it runs an important Eating Disorder program. Literature on the topic usually states that drug use is a much more common issue among boys. However, we consider that this issue is not a major limitation in terms of exploring the efficacy of the intervention since both the experimental and the TAU group in this sample presented a similar gender distribution. Yet, it could limit the generalization of our results to the general population.

In our study, both groups received a standard care treatment program throughout the observation period, and it is unlikely that this standard care, although not specifically intended to assess and treat substance-use related problems, did not have an effect on the probands. At the time of developing the present study, no specific treatment program for substance-use among adolescents attending our department was offered. Thus, diagnosis and treatment of comorbid substance-use was delivered by the professionals in the treatment setting. Professionals were aware of the inclusion of patients in our trial, and were told not to change their approach to substance-use. Hence, we must consider that throughout the observation period, the probands in both the TAU and the intervention groups experienced an intervention for their primary psychiatric condition and a subsequent, non-controlled intervention for their substance-use, which might have influenced the study outcomes. Nevertheless, participants in both groups only showed changes over time in their knowledge about psychoactive substances, without changes in intention to use and the other variables. Thus, we can conclude that the standard treatment program as a whole only had a subtle effect on the substance-use of the patients. On the other hand, changes in overall knowledge differed between the two groups, being specifically more intense among the patients who received the brief intervention. And patients in the intervention group showed a change across time in perception of risk (although this was not significant when compared to the TAU group).

\section{Conclusions}

An important conclusion that can be drawn from these results is that implementation of specific targeted programs among this population is an urgent need, since standard care programs seem to not succeed in significantly changing attitudes towards substance-use in adolescents, particularly among those already showing substance-use problems. Brief interventions have an effect on the probands, although a minor one, and probably need to be more intense or qualitatively different in this specific population.

Acknowledgments This study has been supported by a grant from the INIFD (National Institute for Research and Training on Drugs) (INT/1525/2003) as part of the Spanish Government's National Plan on Drugs (Ministry of Health and Consumption).

\section{References}

1. American Psychiatric Association (1994) Diagnostic and statistical manual of mental disorders, 4th edn. American Psychiatric Press, Washington, DC

2. Baker A et al (2002) Evaluation of a motivational interview for substance use within psychiatric in-patient services. Addiction 97(10):1329-1337

3. Barnett NP et al (2003) Identification and brief treatment of alcohol problems with medical patients: an international perspective. Alcohol Clin Exp Res 27(2):262-270

4. Beich A et al (2002) Screening and brief intervention for excessive alcohol use: qualitative interview study of the experiences of general practitioners. BMJ 325(7369):870

5. Bien TH, Miller WR, Tonigan JS (1993) Brief interventions for alcohol problems: a review. Addiction 88(3):315-335

6. Couwenbergh C et al (2006) Comorbid psychopathology in adolescents and young adults treated for substance use disorders. Eur Child Adolesc Psychiatry 15(6):319-328

7. Díaz R et al (2008) Clinical and research utility of Spanish TeenAddiction Severity Index (T-ASI). Addict Behav 33(1):188-195

8. Dunn C, Deroo L, Rivara FP (2001) The use of brief interventions adapted from motivational interviewing across behavioral domains: a systematic review. Addiction 96(12):1725-1742 
9. Fulkerson JA, Harrison PA, Beebe TJ (1999) DSM-IV substance abuse and dependence: are there really two dimensions of substance use disorders in adolescents? Addiction 94(4):495-506

10. Grenard JL et al (2007) Brief intervention for substance use among at-risk adolescents: a pilot study. J Adolesc Health 40(2):188-191

11. Hesselbrock $M$ et al (1999) A validity study of the SSAGA-a comparison with the SCAN. Addiction 94(9):1361-1370

12. Kaminer Y, Bukstein O, Tarter RE (1991) The Teen-Addiction Severity Index: rationale and reliability. Int J Addict 26(2):219 226

13. Knight JR et al (2005) Motivational interviewing for adolescent substance use: a pilot study. J Adolesc Health 37(2):167-169

14. Madrid Anti drug Agency (2003) Spanish Version of the Evaluation Instruments Bank (EIB). Banco de Instrumentos para la Evaluación de Intervenciones preventivas (BIP). Comunidad de Madrid, Madrid

15. McCambridge J, Strang J (2003) Development of a structured generic drug intervention model for public health purposes: a brief application of motivational interviewing with young people. Drug Alcohol Rev 22(4):391-399

16. McCambridge J, Strang J (2004) The efficacy of single-session motivational interviewing in reducing drug consumption and perceptions of drug-related risk and harm among young people: results from a multi-site cluster randomized trial. Addiction 99(1):39-52

17. Migneault JP, Pallonen UE, Velicer WF (1997) Decisional balance and stage of change for adolescent drinking. Addict Behav 22(3):339-351

18. Miller WR (1991) Motivational interviewing: preparing people to change addictive behaviour. Guilford Press, New York

19. de la Moral Jiménez M et al (2006) Factors related to young people's attitudes to the consumption of alcohol and other psychoactive substances. Psicothema 18(1):52-58

20. Moyer A et al (2002) Brief interventions for alcohol problems: a meta-analytic review of controlled investigations in treatmentseeking and non-treatment-seeking populations. Addiction 97(3):279-292
21. Niethammer O, Reiner F (2007) Prevalence of use, abuse and dependence on legal and illegal psychotropic substances in an adolescent inpatient psychiatric population. Eur Child Adolesc Psychiatry 16(4):254-259

22. O'Donnell D et al (1998) Informativeness of child and parent reports on substance use disorders in a sample of ADHD probands, control probands, and their siblings. J Am Acad Child Adolesc Psychiatry 37:752-758

23. Rey JM (2008) Marijuana use disorders and DSM-V. J Am Acad Child Adolesc Psychiatry 47(2):121-122

24. Schwartz RH (2002) Marijuana: a decade and a half later, still a crude drug with underappreciated toxicity. Pediatrics 109(2):284 289

25. Shakeshaft AP et al (2002) Community-based alcohol counselling: a randomized clinical trial. Addiction 97(11):1449-1463

26. Spanish Drug Observatory and Spanish, 2004 (2004) Annual inform from the Spanish Drug Observatory and Spanish Government Delegation for the National Plan on Drugs (Ministry of Health and Consumer Affairs) resources page. National Plan on Drugs web site. Available at http://www.pnsd.msc.es/Categoria2/ publica/pdf/oed-2004.pdf. Accessed 13 March 2007

27. Spirito A et al (2004) A randomized clinical trial of a brief motivational intervention for alcohol-positive adolescents treated in an emergency department. J Pediatr 145(3):396-402

28. Tait RJ, Hulse GK, Robertson SI (2004) Effectiveness of a briefintervention and continuity of care in enhancing attendance for treatment by adolescent substance users. Drug Alcohol Depend 74(3):289-296

29. Tobler NS (1997) Meta-analysis of adolescent drug prevention programs: results of the 1993 meta-analysis. NIDA Res Monogr 170:5-68

30. Trujillo AM et al (2007) Substance use and risk perception: comparative study of adolescents in Bogotá and Barcelona. Adicciones 19(2):179-189

31. Vasilaki EI, Hosier SG, Cox WM (2006) The efficacy of motivational interviewing as a brief intervention for excessive drinking: a meta-analytic review. Alcohol 41(3):328-335 